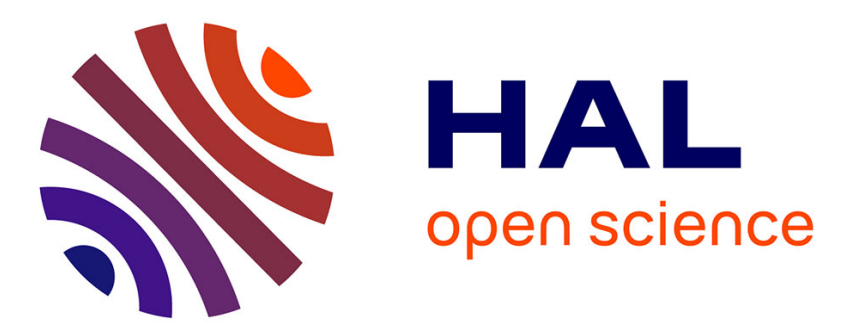

\title{
On the use of solid-shell elements for thin structures: Application to impact and sheet metal forming simulations
}

\author{
Peng Wang, Hocine Chalal, Farid Abed-Meraim
}

\section{- To cite this version:}

Peng Wang, Hocine Chalal, Farid Abed-Meraim. On the use of solid-shell elements for thin structures: Application to impact and sheet metal forming simulations. 19th International ESAFORM Conference on Material Forming (ESAFORM 2016), Apr 2016, Nantes, France. 10.1063/1.4963460 . hal-03246834

\section{HAL Id: hal-03246834 \\ https://hal.science/hal-03246834}

Submitted on 2 Jun 2021

HAL is a multi-disciplinary open access archive for the deposit and dissemination of scientific research documents, whether they are published or not. The documents may come from teaching and research institutions in France or abroad, or from public or private research centers.
L'archive ouverte pluridisciplinaire HAL, est destinée au dépôt et à la diffusion de documents scientifiques de niveau recherche, publiés ou non, émanant des établissements d'enseignement et de recherche français ou étrangers, des laboratoires publics ou privés. 


\title{
On the Use of Solid-Shell Elements for Thin Structures: Application to Impact and Sheet Metal Forming Simulations
}

\author{
Peng WANG $^{\text {a) }}$, Hocine CHALAL ${ }^{\text {b) }}$ and Farid ABED-MERAIM ${ }^{\mathrm{c})}$ \\ LEM3, UMR CNRS 7239 - Arts et Métiers ParisTech, 4, rue Augustin Fresnel, 57078 Metz Cedex 03, France \\ ${ }^{a)}$ Corresponding author: peng.wang@ensam.eu \\ b)hocine.chalal@ensam.eu \\ c) farid.abed-meraim@ensam.eu
}

\begin{abstract}
A family of linear and quadratic assumed-strain based solid-shell elements (SHB) is presented in this paper to simulate 3D thin structural problems including both quasi-static and dynamic analyses. The SHB solid-shell elements are based on a three-dimensional formulation, with only displacements as degrees of freedom, and a reduced integration technique with an arbitrary number of integration points along the thickness direction, which enables them to model 3D thin structures with only one layer of elements through the thickness. All SHB elements have been successfully implemented into ABAQUS dynamic/explicit and static/implicit codes. Several static and dynamic benchmark tests as well as sheet metal forming process simulations, involving large strain, material nonlinearity and contact, have been conducted to assess the performance of the SHB elements.
\end{abstract}

\section{INTRODUCTION}

In nowadays manufacturing industry, thin structures are indispensably designed and employed to reduce weight of products, while improving their mechanical performances (strength, crashworthiness ...). The simulation of these thin structures using the finite element method has become more and more important in the design and manufacture processes. However, due to the large length/thickness ratio, which characterizes thin structures, the traditional solid and shell elements suffer from various locking phenomena. Thus, new types of finite elements are necessary to be established to guarantee a reliable description of nonlinear phenomena through the thickness. In the past two decades, the concept of "solid-shell elements" attracted much attention due to their advantages compared to traditional solid and shell elements. Generally, solid-shell elements are based on a fully three-dimensional formulation, using only displacements as degrees of freedom, and a reduced integration scheme combined with enhanced assumed strain (EAS) methods (see, e.g., [1-5]). In this contribution, a family of assumed-strain based solid-shell (SHB) elements is briefly introduced. It consists of two linear eight-node hexahedral (SHB8PS) and six-node prismatic (SHB6) elements, and their quadratic counterparts (SHB20 and SHB15, respectively). All SHB elements are implemented into ABAQUS dynamic/explicit and static/implicit software packages in order to simulate 3D thin structural problems. In this paper, the general formulation of the SHB solid-shell elements is first briefly introduced. Then, several nonlinear benchmark problems, including impact problem and sheet metal forming process simulations are conducted to assess the performance of the SHB elements when large strain, material nonlinearity and contact are considered.

\section{SHB ELEMENT FORMULATION}

In this section, the basic formulation of the SHB elements is summarized. The detailed formulation of each element can be found in the previous works $[3,4,6-8]$. 


\section{Geometry and Integration Points}

Figure 1 shows the geometry and location of integration points for the SHB elements. In the latter, the special direction $\zeta$ is chosen as the thickness direction, along which an arbitrary number of integration points are distributed. In general, only two integration points through the thickness are sufficient to model most elastic problems, while five integration points are recommended [4] to obtain accurate solutions for nonlinear problems involving material nonlinearities (e.g., plasticity).

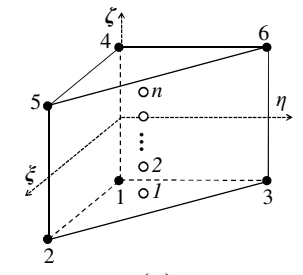

(a)

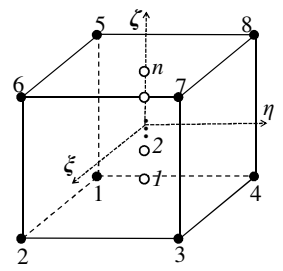

(b)

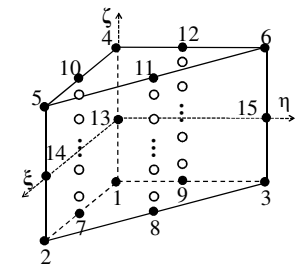

(c)

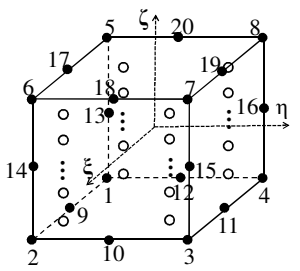

(d)

FIGURE 1. Reference geometry of the SHB elements and location of their integration points:(a) 6-node prismatic SHB6, (b) 8-node hexahedral SHB8PS, (c) 15-node prismatic SHB15, and (d) 20-node hexahedral SHB20

\section{General Formulation of the Dynamic Versions}

The SHB solid-shell elements are based on a fully 3D formulation, as used for traditional solid elements. Accordingly, their isoparametric shape functions are the same as those of standard linear and quadratic solid elements. The coordinates $x_{i}$ and velocities $v_{i}$ inside each SHB element are interpolated using the corresponding nodal variables as follows:

$$
\begin{gathered}
x_{i}=x_{i I} N_{I}(\xi, \eta, \zeta)=\sum_{I}^{n} N_{I}(\xi, \eta, \zeta) x_{i I} \\
v_{i}=\dot{d}_{i I} N_{I}(\xi, \eta, \zeta)
\end{gathered}
$$

where $x_{i I}$ and $\dot{d}_{i I}$ represent the nodal coordinates and the nodal velocities, respectively. The lowercase subscript $i$ varies from 1 to 3 and represents the spatial coordinate directions; the uppercase subscript $I$ represents the number of element nodes.

Using the above interpolation, the velocity gradient $\nabla_{s}(\mathbf{v})$ is related to the nodal velocity field $\mathbf{d}$ by the following relationship:

$$
\nabla_{s}(\mathbf{v})=\mathbf{B} \cdot \dot{\mathbf{d}}
$$

where $\mathbf{B}$ is the discrete gradient operator. Then, the so-called assumed-strain method is used in the formulation of the SHB solid-shell elements, which is based on the simplified form of the Hu-Washizu principle [9]

$$
\pi(\dot{\overline{\boldsymbol{\varepsilon}}})=\int_{\Omega_{e}} \delta \dot{\bar{\varepsilon}}^{T} \cdot \boldsymbol{\sigma} d \Omega-\delta \dot{\mathbf{d}}^{T} \cdot \mathbf{f}^{e x t}=0
$$

where $\delta$ represents a variation, $\dot{\overline{\boldsymbol{\varepsilon}}}$ the assumed-strain rate, $\boldsymbol{\sigma}$ the stress field, $\dot{\mathbf{d}}$ the nodal velocities, and $\mathbf{f}^{\text {ext }}$ the external nodal forces. The assumed-strain rate $\dot{\overline{\boldsymbol{\varepsilon}}}$ is expressed in terms of a new matrix $\overline{\mathbf{B}}$, which is projected from the classic discrete gradient operator defined in Eq. 3 


$$
\dot{\overline{\mathbf{\varepsilon}}}(x, t)=\overline{\mathbf{B}}(x) \cdot \dot{\mathbf{d}}(t)
$$

Substituting the above equation into the simplified form of the $\mathrm{Hu}-$ Washizu principle, the internal force $\mathbf{f}^{\text {int }}$ can be derived as

$$
\mathbf{f}^{\text {int }}=\int_{\Omega_{e}} \overline{\mathbf{B}}^{T} \cdot \boldsymbol{\sigma} d \Omega
$$

Additionally, for dynamic analysis, an initial mass matrix is required in the formulation of the SHB elements. In the latter, the same lumped mass matrices as those used for standard three-dimensional elements are adopted for the SHB elements.

\section{General Formulation of the Static Versions}

The general formulation of the static versions of the SHB elements is similar to the above dynamic one, and will not be repeated here. Instead of the mass matrix in dynamic analysis, the stiffness matrix $\mathbf{K}_{e}$ is required in static analysis, which is also derived from the simplified form of the $\mathrm{Hu}-\mathrm{Washizu}$ principle as follows:

$$
\mathbf{K}_{e}=\int_{\Omega_{e}} \overline{\mathbf{B}}^{T} \cdot \mathbf{C}^{e p} \cdot \overline{\mathbf{B}} d \Omega+\mathbf{K}_{G E O M}
$$

where the geometric stiffness matrix $\mathbf{K}_{G E O M}$ is due to the nonlinear (quadratic) part of the strain [4].

In addition, special treatments, such as the stabilization technique for the linear SHB8PS element and the projection technique for the linear SHB6 and SHB8PS elements, are applied in order to avoid all locking phenomena and to guarantee the accuracy of the solution (the full details on these techniques can be found in [3,4,6-8]).

\section{NUMERICAL EXAMPLES AND DISCUSSIONS}

In this section, several numerical examples are presented to assess the performance of the SHB solid-shell elements in both quasi-static and dynamic analysis. As mentioned in the above sections, an arbitrary number of integration points can be used with the SHB solid-shell elements. In the following benchmark tests, only two integration points through the thickness are used, which is sufficient to obtain accurate solutions, while five integration points are required to obtain accurate predictions in the simulation of the deep drawing test.

\section{Static Analysis of a Cantilever Plate}

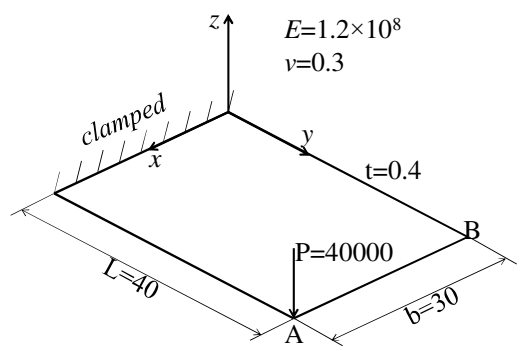

(a)

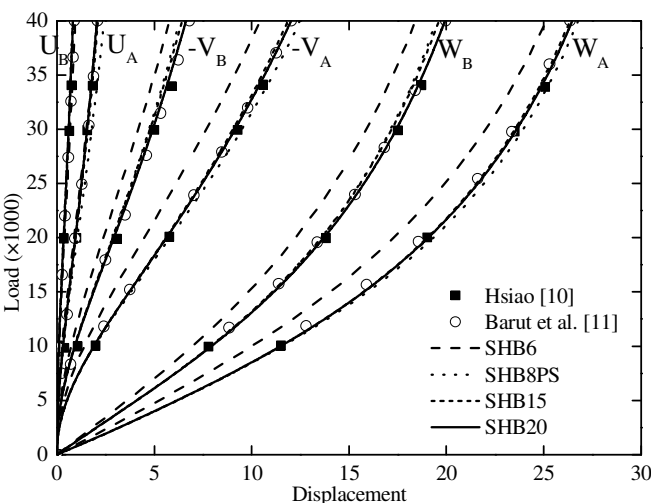

(b)

FIGURE 2. Simulation of rectangular cantilever plate: (a) Geometry and material parameters; (b) Load-displacement results 
An elastic cantilever plate with a concentrated force at one corner, as proposed by Hsiao [10], is selected here to evaluate the SHB solid-shell elements in large displacements and rotations. The corresponding geometric parameters as well as the elastic properties are summarized in Fig. 2 (a). The load-displacement curves at the corner points A and B (as depicted in Fig. 2 (a)), are shown in Fig. 2 (b). Compared with the reference results given by Hsiao [10] and Barut et al. [11], the predicted results using the SHB elements are in good agreement, except for the linear prismatic SHB6 element, for which a finer mesh is required to obtain an accurate solution.

\section{Dynamic Analysis of a Simply Supported Square Plate}

Figure 3 illustrates a popular dynamic benchmark test for finite element analysis, which was studied by many researchers [12-14]. As shown in Fig. 3 (a), a simply supported elastic-plastic square plate is subjected to a uniform pressure. Owing to the symmetry of the problem, only one quarter of the plate is modeled using SHB elements. The predicted central vertical displacement history is chosen to evaluate the respective performance of the SHB elements. All predicted results with the SHB elements are reported in Fig. 3 (b) along with the reference solutions obtained from [12-14]. It can be seen that both the maximum displacement and the time period obtained with the SHB elements are well predicted with respect to the reference solutions.

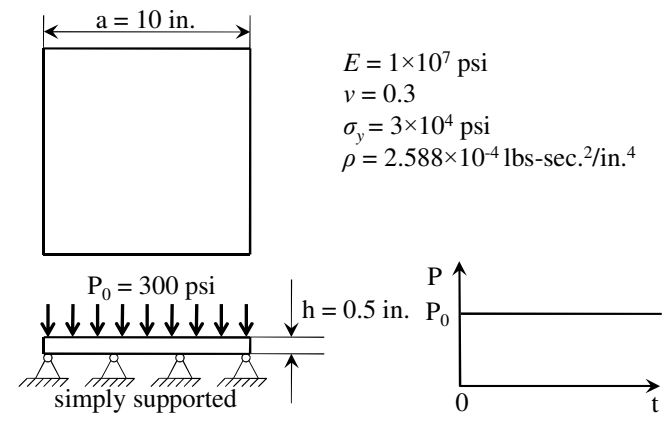

(a)

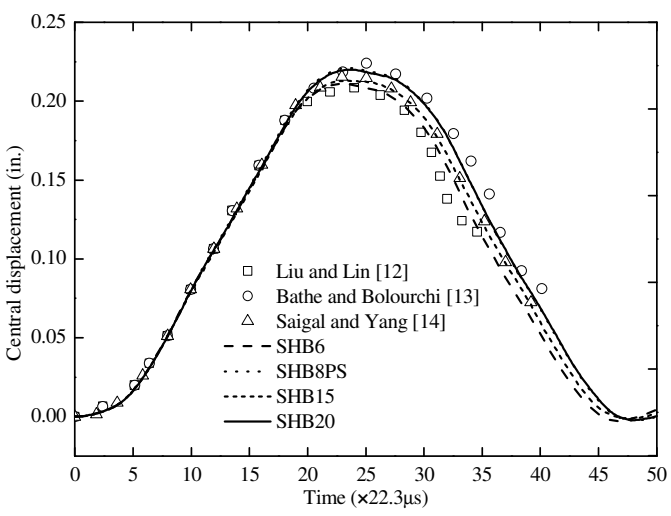

(b)

FIGURE 3. Simply supported square plate subjected to a uniform pressure: (a) Geometry and material parameters; (b) Central displacement history results

\section{Low Velocity Impact Problem}

The dynamic response of a clamped circular plate subjected to an impact by a projectile, as studied by Chen and Yang [15], is considered here to assess the capabilities of the SHB elements in handling elastic-perfectly-plastic impact-contact problems. The geometric dimensions and boundary conditions of the plate and the impactor are depicted in Fig. 4. The impactor is modeled as a rigid body with an assigned mass, while the circular plate is made of 6061-T6 aluminum alloy with the following material properties: Young's modulus $E=69 \mathrm{GPa}$, Poisson's ratio $v=0.3$, yield stress $\sigma_{0}=290 \mathrm{MPa}$ and density $\rho=2600 \mathrm{Kg} / \mathrm{m}^{3}$. The frictionless hard-contact is defined between the plate and the impactor. Two typical cases with different initial mass and velocity of the impactor are considered: Case 1: $\mathrm{M}=23.5 \mathrm{~g}, \mathrm{~V}_{0}=49.1 \mathrm{~m} / \mathrm{s}$ and Case $2: \mathrm{M}=54.4 \mathrm{~g}, \mathrm{~V}_{0}=29.9 \mathrm{~m} / \mathrm{s}$.

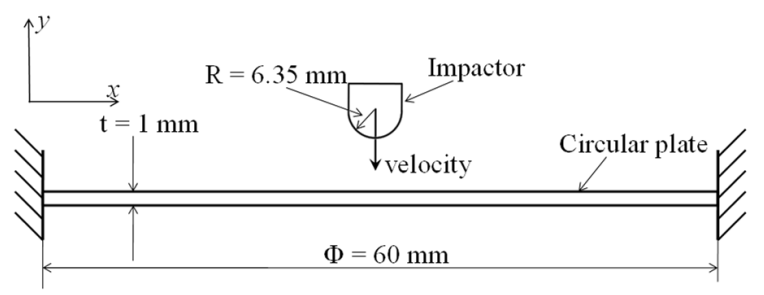

FIGURE 4. Geometry and boundary conditions of the impact problem 
Owing to the symmetry of the problem, only one quarter of the plate is modeled with a single element layer of 2708 elements in the case of the linear prismatic SHB6 element, 934 elements with the linear hexahedral SHB8PS element, 926 elements with the quadratic prismatic SHB15 element, and 463 elements in the case of the quadratic hexahedral SHB20 element (see Fig. 5). Figures 6 (a) and 6 (b) show the predicted dynamic response for the impact force corresponding to cases 1 and 2, respectively, along with the reference numerical solutions provided by Chen and Yang [15]. It can be seen that the impact force history predicted with the SHB elements is well reproduced when compared to the reference solutions for both studied cases. More specifically, the double impact force peak of the dynamic solutions, which is typically observed in such impact problems, as well as the order of magnitude of the maximum impact force peak, corresponding to the end of the indentation stage, are in good agreement with the reference solutions.

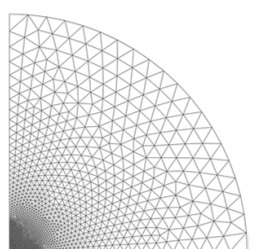

(a) SHB6

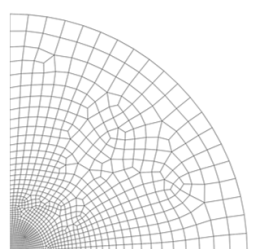

(b) SHB8PS

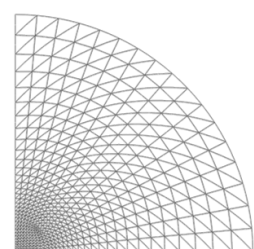

(c) SHB15

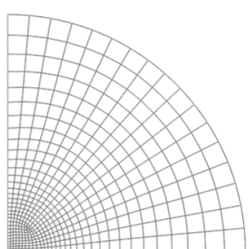

(d) SHB20

FIGURE 5. Initial mesh of the circular plate

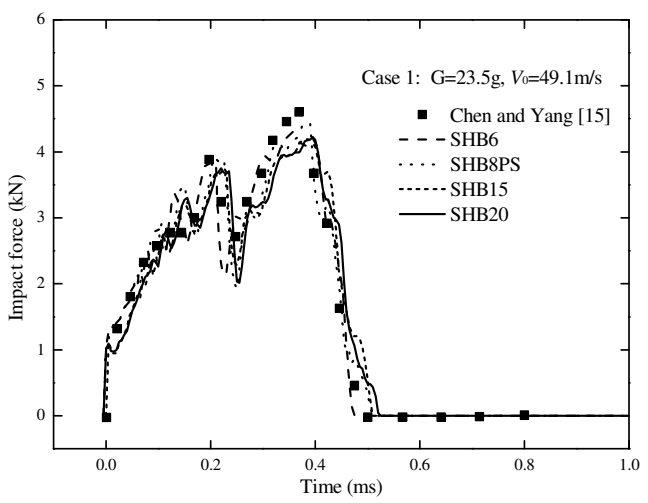

(a)

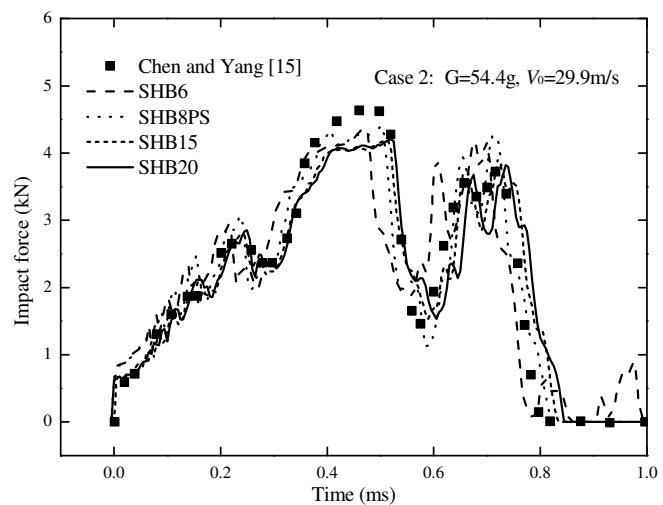

(b)

FIGURE 6. Impact force history predicted by the SHB elements along with the reference solutions: (a) Case 1 and (b) Case 2

\section{Deep Drawing of a Rectangular Cup}

Deep drawing of a rectangular cup, as proposed by Choi and Huh [16], is conducted here to evaluate the performance of the SHB elements in the context of sheet metal forming simulation. An initial rectangular steel sheet, with dimensions of $120 \mathrm{~mm} \times 170 \mathrm{~mm} \times 0.625 \mathrm{~mm}$, is modeled with the Swift isotropic hardening law and the anisotropic Hill' 48 yield criterion. Owing to the symmetry, only one quarter of the model is discretized by the SHB elements. The final deformed meshes of the full cup obtained with the SHB elements are presented in Fig. 7.

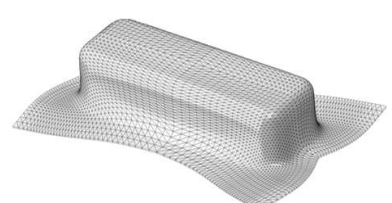

(a) SHB6

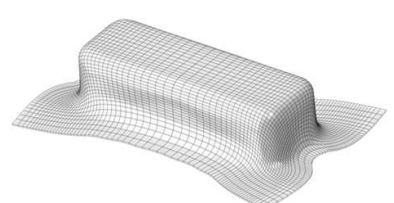

(b) SHB8PS

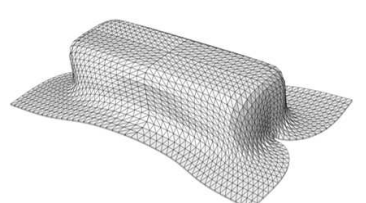

(c) SHB15

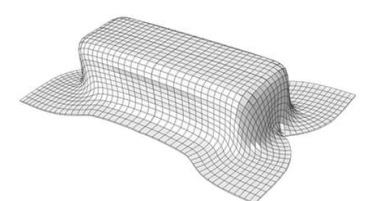

(d) SHB20

FIGURE 7. Final deformed mesh of the rectangular cup

As shown in Fig. 7, the deep drawing of the rectangular cup involves extremly large strain in the whole sheet, and especially the width cup region, where severe distortions due to strong in-plane and through the thickness 
nonlinearities could cause convergence issues. In such situations, the use of the dynamic versions of the SHB solidshell elements with 5 integration points along the thickness is recommended. In Fig. 8, the final simulated flange contours at different drawing stages (punch stroke $=10 \mathrm{~mm}, 20 \mathrm{~mm}$, and $30 \mathrm{~mm}$ ). In the first two stages, one can note that, generally, the flange contours obtained with the SHB elements are better than those predicted by the reducedintegration shell element (S4R) and solid-shell elements (SC8R and SC6R) available in ABAQUS code compared to the experimental measurements reported in [16]. While when the punch moves deeper (punch stroke $=30 \mathrm{~mm}$ ), very large strain and rotation occur in the short-width cup region, which results in irresistible mesh distotions for solid-like elements. Comparing to the experimental result, the SHB8PS element predict the final flange contour more accurate than other SHB elements and even ABAQUS shell or solid-shell elements, which can be viewed intuitively by the final deformed cup shapes shown in Figure 7.

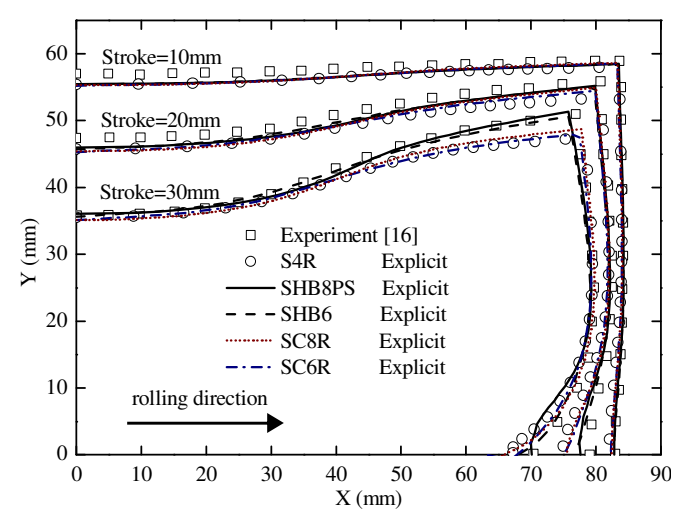

(a)

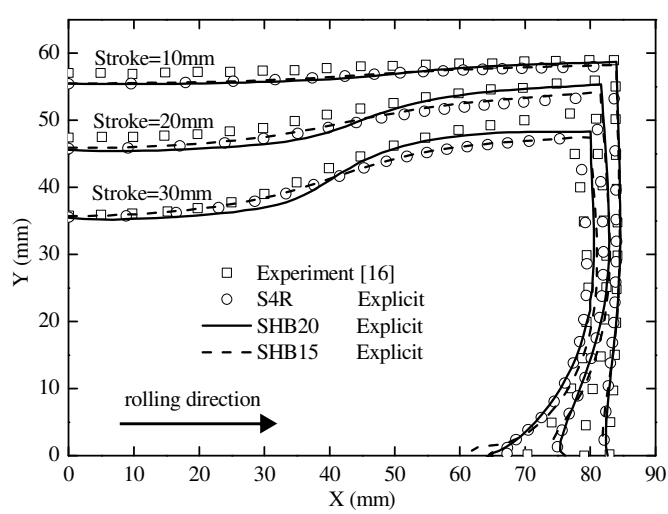

(b)

FIGURE 8. Flange contours of the rectangular cup predicted with (a) linear SHB elements, and (b) quadratic SHB elements

\section{CONCLUSIONS}

In the present work, a family of linear and quadratic solid-shell elements is proposed to model 3D thin structural problems. All of these elements have been successfully implemented into ABAQUS static/implicit and dynamic/explicit codes. Their implementation and respective performance have been validated first on two nonlinear numerical benchmarks and on an impact test problem. Then, the proposed elements have been used in the simulation of deep drawing of a rectangular cup, through which the SHB elements demonstrated their capabilities of accurately describing the various nonlinear through-thickness phenomena with only a single element layer.

\section{REFERENCES}

1. R. Hauptmann and K. Schweizerhof, Inter. Jour. Numer. Meth. Engng. 42, 49-70 (1998).

2. S. Klinkel et al., Computers \& Structures 71, 43-62 (1999).

3. F. Abed-Meraim and A. Combescure, Computers \& Structures 80, 791-803 (2002).

4. F. Abed-Meraim and A. Combescure, Inter. Jour. Numer. Meth. Engng. 80, 1640-1686 (2009).

5. M. Pagani et al., Comput. Methods Appl. Mech. Engng. 268, 141-159 (2014).

6. V. D. Trinh et al., J. Mech. Sci. Technol. 25, 2345-2364 (2011).

7. A. Salahouelhadj et al., Arch. Appl. Mech. 82, 1269-1290 (2012).

8. F. Abed-Meraim et al., Computing 95, 373-394 (2013).

9. J. C. Simo and T. J. R. Hughes, Jour. Appl. Mech. 53, 51-54 (1986).

10. K. M. Hsiao, Comput. Struct. 25, 655-675 (1987).

11. A. Barut et al., Comput. Methods Appl. Mech. Engng. 143, 155-173 (1997).

12. S. C. Liu and T. H. Lin, Earthq. Eng. Struct. Dyn. 7, 147-159 (1979).

13. K. J. Bathe and S. Bolourchi, Computers \& Structures 11, 23-48 (1980).

14. S. Saigal and T. Y. Yang, Inter. Jour. Numer. Meth. Engng. 21, 1115-1128 (1985).

15. L. B. Chen and J. L. Yang, Acta Mech. Sin. 23, 415-425 (2007). 
16. T. H. Choi and H. Huh, J. Mater. Process. Tech. 89-90, 58-64(1999). 